De l'accès aux soins aux « trajectoires du mourir ». Les étrangers atteints de cancer face aux contraintes administratives

From Health Care Access to "End-of-life Trajectories". Foreign Cancer Patients Facing Administrative Obstacles

Desde el acceso a la salud hasta las «trayectorias del morir»: los extranjeros enfermos de cáncer frente a los requerimientos administrativos

\title{
Anaïk Pian
}

\section{(2) OpenEdition}

\section{Journals}

Édition électronique

URL : https://journals.openedition.org/remi/5915

DOI : 10.4000/remi.5915

ISSN : $1777-5418$

\section{Éditeur}

Université de Poitiers

\section{Édition imprimée}

Date de publication : 1 juin 2012

Pagination : 101-127

ISBN : 979-10-90426-04-7

ISSN : 0765-0752

\section{Référence électronique}

Anaïk Pian, « De l'accès aux soins aux « trajectoires du mourir ». Les étrangers atteints de cancer face aux contraintes administratives ", Revue européenne des migrations internationales [En ligne], vol. 28 $n^{\circ} 2$ | 2012, mis en ligne le 10 octobre 2012, consulté le 14 avril 2022. URL : http:// journals.openedition.org/remi/5915; DOI : https://doi.org/10.4000/remi.5915 


\section{De l'accès aux soins aux « trajectoires du mourir $\gg$. Les étrangers atteints de cancer face aux contraintes administratives}

\section{Anaik PIAN ${ }^{1}$}

Z $\mathrm{n}$ France, certaines maladies, comme le cancer, demeurent un sujet très peu tantes concernent en premier lieu d'autres pathologies comme le VIH-sida ou la tuberculose (Cognet, Bascougnano et Adam-Veniza, 2009), les troubles psychiatriques des demandeurs d'asile (Pestre, 2010) à moins qu'elles ne portent sur l'accès aux soins en général (Fassin et al., 2001 ; Aeberhard et Lebas, 2011) ou les initiatives locales mises en œuvre pour la prise en charge sanitaire et sociale de cette population (Hoyez, 2011).

Certes, des recherches sur les cancers professionnels mentionnent le cas de travailleurs immigrés exposés à des produits cancérigènes au cours de carrières précaires et ayant des difficultés à faire reconnaître l'origine professionnelle de leur maladie (Lanna, Marchand et Thébaud-Mony, 2000 ; Boujasson, 2009). D'autres études se sont intéressées à la prévention des cancers féminins et aux pratiques de dépistage des femmes d'origine étrangère (Grilo, Soler et Chauvin, 2012). Toutefois, les travaux en sciences sociales sur le cancer des étrangers et des immigrés demeurent très peu développés (Chaouchi, Casu et Ledesert, 2006) et la plupart des études réalisées relèvent d'approches épidémiologiques ou ethnopsychiatriques, souvent culturalistes ou essentialisantes. Une brève revue de cette littérature apparaît alors nécessaire afin de mieux situer, par la suite, la perspective qui est la nôtre.

Les études épidémiologiques cherchent à mesurer et à rendre compte des risques de cancer et de la mortalité cancéreuse selon l'origine ethnique. Les travaux de Brahimi (1980) qui mettent en évidence, dès les années 1980, la sous-mortalité par cancer des étrangers en France sont suivis dans les années 1990, d'une série d'études sur la mortalité par cancer des Chinois et des immigrés originaires du Sud-est asiatique (Bouchardy, Parkin et Khlat, 1994), d'Afrique subsaharienne (Bouchardy et al., 1995) ou encore d'Afrique du Nord (Bouchardy et al., 1996). Outre une discussion sur d'éventuels facteurs géné-

1 Post-doctorante en sociologie, Université de Bretagne Occidentale (ARS), 20 avenue Duquesne, 29200 Brest ; anaikpian@yahoo.fr 
tiques, des hypothèses mettant en avant l'impact de comportements sociaux différenciés (fécondité, habitudes alimentaires, emplois occupés, etc.) sont proposées pour expliquer cette sous-mortalité (Darmon et Khlat, 2001). Celle-ci est également envisagée à partir des biais pouvant provenir des effets de sélection de la migration (healthy migrant affect), de la structure démographique de l'immigration ainsi que des retours au pays d'origine des immigrés malades et en fin de vie ; les décès à l'étranger n'étant pas comptabilisés dans les registres d'états civils français (Khlat, Sermet et Laurier, 1998). Cependant, les études épidémiologiques se basent sur les données issues des recensements de la population et, de ce fait, toute une catégorie d'étrangers (sans-papiers, titulaires de visa touristique, etc.) échappe en partie à ces statistiques.

D'autres travaux, s'inscrivant dans des approches transculturelles, d'ethnopsychiatrie ou d'ethnomédecine, s'orientent vers des thématiques récurrentes, telles les différences culturelles, la relation soignant-soigné ou les représentations de la maladie. L'expérience de la maladie et sa mise en récit sont analysées en regard de modèles culturels associés à l'origine ethnique des individus (Ibrahima, 2009 ; Koné, 2010). Inscrites dans une perspective culturaliste, ces études tendent à réduire les immigrés et les étrangers à leur culture, dite culture d'origine, considérée en elle-même comme le facteur explicatif des comportements individuels et collectifs et notamment des difficultés rencontrées dans la confrontation au monde médical. Ces recherches reposent sur une conception essentialisante de la figure de l'Autre, sans que soient prises en considération la dynamique des rapports interethniques, les conditions de la migration, ni même les contraintes institutionnelles des contextes de soin.

Cet article se démarque de ces approches en se situant dans une perspective qualitative à la croisée d'une sociologie des migrations et d'une sociologie de la santé. Il porte un regard sur deux moments clés (pouvant être plus ou moins distants dans le temps) de la confrontation des étrangers atteints de cancer à l'espace du soin : d'une part, il s'intéresse aux configurations d'entrée dans l'espace du soin ; d'autre part, il s'intéresse aux «trajectoires du mourir » dès lors qu'un échec thérapeutique est annoncé.

Cette mise en miroir est intéressante à double titre. D’un point de vue empirique tout d'abord, car si les contraintes (légales) d'accès au soin font partie des données les mieux renseignées dans les recherches portant sur la «santé des migrants », celles pesant sur les « trajectoires du mourir » sont beaucoup moins étudiées. D'un point de vue analytique ensuite, car ce regard porté aux deux extrémités de trajectoires de maladie ${ }^{2}$ permet de faire ressortir, de bout en bout, les logiques des acteurs face aux contraintes institutionnelles et leurs implications, tant sur l'expérience profane du soin que sur les pratiques professionnelles. En effet, de nombreux travaux ont pointé l'impact des facteurs socioéconomiques, de la situation migratoire et des discriminations (directes et indirectes) dans les inégalités d'accès et de recours aux soins des immigrés (Berchet et Jusot, 2012). Pour autant, " tous les problèmes » ne se sont pas résolus une fois ces premières difficultés surmontées. Il importe donc de déplacer et de prolonger la focale au-delà. En l'occurrence, comme nous le verrons, si papiers et couverture médicale sont déterminants dans les modalités d'accès au soin, ils sont tout aussi importants dans les « trajectoires du mourir ».

2 Cela ne suppose pas que l'évolution de ces trajectoires soit linéaire, celles-ci pouvant passer par différentes phases de stabilité, d'amélioration ou de détérioration (Ménoret, 1999). 
Par « trajectoire du mourir », nous faisons référence à la notion de « trajectoire » telle que l'a développée Strauss (1992 : 29) dans ses travaux sur les maladies chroniques. Cette notion rend compte du travail (effectué par les professionnels, mais aussi par les patients et leurs proches) d'organisation du cours de la maladie, tout en s'intéressant aux conséquences de ce travail sur les acteurs impliqués. Ce n'est donc pas seulement l'évolution de la pathologie qui est prise en considération ni le sens donné à la maladie, mais l'ensemble des actions et des pratiques qui mettent en forme sa " gestion ». Le terme de « trajectoire de mourir » s'inscrit dans le prolongement de ce modèle d'analyse tout en se rapportant à la phase ultime de la fin de vie (Castra, 2003).

L'espace du soin, dans lequel nous replaçons ces trajectoires, est également à définir, car il fait l'objet de différentes conceptualisations en sciences sociales. Fassin (1991 : 58) l'appréhende comme « un produit du monde social » mettant « en relation un ensemble d'agents qui s'y rencontrent, comme malades, professionnels, administrateurs » et qui « prend son sens par rapport à la manière dont s'y inscrit le politique » (ibid. : 16). Cette définition socio-anthropologique met au premier plan de l'analyse les rapports de force constitutifs de cet espace. Des géographes, en revanche, mettent l'accent sur l'inscription physique de l'espace du soin, en tant que lieu (hôpital, domicile, etc.) où s'exercent des pratiques profanes et professionnelles, même si les frontières de cet espace (entre le « dedans » et le « dehors ») peuvent être plus ou moins floues. Le rapport à l'espace est également interrogé à travers les significations qu'il revêt pour les différents acteurs et son impact sur l'état de santé et l'expérience de la maladie (Dyck et O'Brien, 2003 ; Curtis et al., 2009). En partant de ces différentes approches, nous proposons une définition opérationnelle de l'espace du soin désignant une configuration socio-spatiale traversée par des rapports sociaux inégalitaires (incluant les rapports interethniques), où les structures institutionnelles pèsent sur les logiques des acteurs (profanes et professionnels) tout en donnant lieu à diverses formes de négociation, de contournement voire de transgression. D'une part, ces dynamiques prennent place dans des lieux précis (centres de soins, services hospitaliers, etc.), porteurs de sens et de normes. D'autre part, elles mettent en jeu différentes échelles de territoire, du local à l'international et au transnational, dans le cadre de trajectoires de maladie (et de mourir) pouvant impliquer plusieurs pays (et notamment ici pays d'immigration et d'origine).

Il s'agira donc, dans cet article, de mettre à jour les contraintes administratives auxquelles se heurtent les étrangers (précaires) atteints de cancer, en les replaçant dans le contexte structurel pesant en amont comme en aval sur les trajectoires de maladie et où se rencontrent politiques d'immigration et contraintes organisationnelles et financières des établissements de santé ${ }^{3}$. Nous montrerons comment, pour les professionnels, la gestion de ces trajectoires interroge à la fois des routines, des normes et des règles organisationnelles, notamment dans des contextes locaux peu préparés à ces nouveaux profils de patients.

3 Cet article est basé sur les résultats d'une recherche subventionnée par l'Institut National du cancer dans le cadre du programme INVUSO : Trajectoires des malades, contextes de soin et inégalités sociales. Étude croisée des formes de vulnérabilité des patients atteints de cancer en évolution (axe 1, Université de Rennes 2) et des patients immigrés et étrangers en Bretagne (axe 2, Université de Bretagne Occidentale). L'axe 2 est coordonné par Simone Pennec (UBO, ARS). Le coordinateur général est Marcel Calvez (Rennes 2, ESO). Je remercie Simone Pennec, Françoise Leborgne-Uguen et les responsables de la coordination de ce numéro de la REMI, pour leurs remarques et suggestions. 
L'étude s'est déroulée en Bretagne, une région historiquement de faible immigration même si le paysage migratoire est aujourd'hui en recomposition. Les étrangers y représentent moins de $2 \%$ de la population (Regnard, 2009), mais la présence immigrée croît régulièrement depuis cinquante ans et, au début des années 2000, plus de $29 \%$ des immigrés résidant dans la région étaient arrivés de l'étranger entre 1990 et 1999 (Dussud et al., 2004). Les flux actuels, provenant majoritairement de pays de l'ex-bloc soviétique et d'Afrique subsaharienne, tranchent avec les courants migratoires antérieurs ${ }^{4}$ et se caractérisent par une proportion importante de demandeurs d'asile. Selon le dernier rapport d'activité de l'OFPRA (2011: 11), la demande d'asile en Bretagne a augmenté de $49 \%$ entre 2009 et 2010 pour atteindre désormais plus de 1000 nouveaux demandeurs par an avec, pour corollaire, une saturation des dispositifs d'accueil ${ }^{5}$.

\section{Méthodologie d'enquête et population d'étude}

Notre recherche a été réalisée à Rennes, Brest et Quimper, villes situées dans deux départements (Ille-et-Vilaine et Finistère). Elle porte sur une catégorie particulière d'étrangers, ceux arrivés depuis peu sur le territoire français et confrontés, du fait ou non de leur maladie, à des situations socio-économiques et juridiques précaires. Seuls les étrangers hors Union européenne ont été retenus. Parmi les personnes rencontrées, différents profils peuvent être identifiés :

- Des demandeurs d'asile, essentiellement originaires de pays de l'Est (Arménie, Géorgie, Tchétchénie, Russie) et d'Afrique (Centrale et de l'Ouest).

- Des étrangers arrivés avec un visa touristique et dont la plupart proviennent du Maghreb et d'Afrique de l'Ouest. Dans un contexte de durcissement des politiques d'immigration, ceux qui obtiennent ces documents de voyage appartiennent à des catégories sociales relativement aisées. Néanmoins, le coût et la durée des soins en France - particulièrement élevés dans le cadre du cancer - peuvent par la suite les confronter à des situations très difficiles.

- Quelques étrangers arrivés en situation irrégulière. Il s'agit de personnes originaires de pays de l'Est (Géorgie, Arménie) et d'Afrique de l'Ouest et de l'Est.

Nous avons réalisé des entretiens semi-directifs avec une quinzaine de patients correspondant à ces trois profils (sur un total d'une quarantaine de patients et de leurs proches rencontrés). Il s'est agi d'entretiens biographiques remontant à la situation dans le pays d'origine avant la migration. Ces entretiens se sont déroulés lors de plusieurs rencontres et ont généralement été prolongés ou précédés par des discussions plus informelles. Nous avons aussi mené des entretiens (enregistrés ou non, semi-directifs ou sous une forme plus ouverte) avec une quarantaine de professionnels des secteurs médical, social et administratif dont le témoignage fait parfois référence à des patients décédés. La combinaison d'entretiens enregistrés et de temps d'échanges moins formels a souvent permis de recueillir, hors micro, des propos plus libres et moins « normés ».

En complément, nous avons effectué quelques observations dans des établissements de soin (vêtue d'une blouse blanche, notre présence a été acceptée dans certains services au côté des soignants). Ces observations ont permis de saisir de manière directe des interactions entre patients et équipes médicales et d'assister aux débats entre professionnels (par exemple dans les salles de pause, lors des transmissions ou dans les cabinets infirmiers) concernant des situations qualifiées de " problématiques ». Par souci d'anonymat, toutes les initiales désignant les patients sont fictives et les pays d'origine des enquêtés ont pu être modifiés dans la présentation des études de cas.

4 Pour plus de précisions sur l'histoire de l'immigration en Bretagne, cf. Morillon et al. (2007). 5 Cf. Ouest France du 15/09/2011 et du 06/12/2010. 
C'est à cette nouvelle donne migratoire que sont confrontés les professionnels des établissements de soin même si, en ce qui concerne la cancérologie, les patients étrangers demeurent proportionnellement peu nombreux.

\section{L'ENTRÉE DANS LE SOIN : ÉTHIQUE, POLITIQUE ET OBSTACLES STRUCTURELS}

L'accès à une couverture maladie est soumis à différentes conditions selon le titre de séjour dont disposent (ou non) les étrangers. Les législations actuelles, qui n'ont eu de cesse de se durcir ces dernières années, sont plus ou moins restrictives et contraignantes selon la situation administrative et l'urgence du soin au regard de l'état de santé6. Sur le terrain, ces législations donnent lieu à des pratiques d'ajustement tant du côté des professionnels des secteurs médicaux et sociaux que des patients. Ce sont ces pratiques que nous chercherons à identifier à travers des cas limites qui révèlent d'une part, les failles du système établi, et d'autre part les logiques des acteurs pour « faire avec » ces contraintes institutionnelles.

\section{Des étrangers malades, des délais administratifs}

L'Aide médicale d'état (AME), créée en 2000, institue un régime spécifique d'affiliation à la sécurité sociale pour les étrangers en situation irrégulière. Jusqu'à une réforme restrictive introduite dans la loi de finances pour $2011^{7}$, trois conditions étaient requises pour en bénéficier : outre l'irrégularité du séjour, il fallait pouvoir justifier de trois mois de présence sur le territoire français et ne pas dépasser un certain plafond de ressources. Ce régime s'applique aux quelques cas rencontrés en Bretagne, d'étrangers atteints de cancer et arrivés sans papiers pour tenter de se faire soigner. L'entrée dans le soin de ces patients s'effectue très souvent dans une forte urgence médicale et sociale, à un stade évolué de la maladie. Madame R., Georgienne, a été recueillie par une équipe du Centre communal d'action sociale (CCAS) alors qu'elle errait devant une gare. Au regard de son état général, elle est adressée aux urgences. L'équipe qui la reçoit, ainsi que l'assistant social de la cellule PASS (Permanence d'accès aux soins) ${ }^{8}$, font très vite appel à une association d'aide aux demandeurs d'asile pour assurer la traduction, la patiente ne parlant pas un mot de français. Cette association est fréquemment appelée par l'hôpital pour assurer la médiation auprès de patients originaires de pays de l'Est, lorsque l'entourage fait défaut et que se pose un problème de langue ${ }^{9}$. Commence alors une course contre la montre : d'une part, pour récupérer les pièces administratives nécessaires à la mise en

6 Cf. l'article de Gabarro dans ce numéro.

7 Cette réforme introduit entre autres, depuis le mois de mars 2011, une réduction du panier de soins et le paiement d'un droit de timbre de trente euros pour les bénéficiaires majeurs. Une première réforme de l'AME en 2003 avait conduit à l'instauration de la conditionnalité de trois mois de présence sur le territoire, qui n'était pas requise dans la loi initiale.

8 Pour plus de précisions sur ces structures, cf. Hachimi-Alaoui et Nacu (2010).

9 Bien qu'il ne soit pas possible de développer ici ce point, les ressources opérationnelles des établissements en termes d'interprétariat sont limitées et des solutions ad hoc sont souvent mises en place pour y remédier. 
place d'une couverture maladie et d'une demande de régularisation pour soins ${ }^{10}$; d'autre part, pour recueillir des informations médicales auprès d'un hôpital en Géorgie, l'équipe ayant appris par l'interprète que la patiente avait reçu un premier traitement anticancéreux dans son pays. L'assistant social fait alors part de la complexité des démarches :

« Elle ne rentrait dans aucun cadre. Elle était sans droits, sans papiers, elle ne pouvait même pas prétendre à l'AME parce qu'il fallait pouvoir dire qu'elle était en France depuis plus de trois mois de façon irrégulière et nous, la seule date qu'on pouvait présenter, c'était celle de l'entrée à l'hôpital. Du coup, on a fait une demande de soins urgents ${ }^{11}$ pour toute cette période-là ».

Dans d'autres cas, ces procédures administratives sont initiées par des centres de soin gratuits, spécialisés ou non dans l'accueil des populations migrantes. En Bretagne en effet, Rennes est la seule ville de la région à disposer d'une structure spécifiquement dédiée aux primo-arrivants. Les étrangers sans-papiers qui s'adressent à ces centres de soin gratuits y sont le plus souvent orientés par les centres sociaux, les hôpitaux, quelques professionnels ou généralistes libéraux, des compatriotes, le réseau de bouche-à-oreille ou des associations communautaires, qu'elles soient de type « interculturel » ou autre (Hoyez, 2011).

La plupart des assistants sociaux hospitaliers rencontrés soulignent que les demandes d'AME qu'ils ont eu à réaliser reçoivent globalement un avis positif et que les exigences des préfectures sont moins drastiques que dans d'autres départements qui, par exemple, refusent les abonnements de bus comme preuve de domiciliation. Comme le soulignent Cognet, Gabarro et Adam-Vezina (2009), mais aussi Fassin et al. (2001), au-delà du cadre juridique, le traitement des dossiers d'AME relève d'une « politique du guichet » faisant l'objet d'interprétations et de pratiques plus ou moins restrictives. Aussi, pour accélérer la mise en place de l'AME face à des cas de cancers très avancés, certains assistants sociaux mobilisent leurs relations interpersonnelles à la Caisse Primaire d'Assurance Maladie (CPAM) et multiplient les appels téléphoniques.

Certains professionnels, relevant du secteur médical ou social, sont néanmoins critiques par rapport aux procédures d'AME. Si le fichage de patients et le risque de

10 Selon la loi du 11 mai 1998 relative à l'entrée et au séjour des étrangers en France et au droit d'asile (CESEDA), les étrangers gravement malades peuvent prétendre à un titre de séjour $\left(\mathrm{L} 313-111^{\circ}\right) .28000$ personnes, soit $0,8 \%$ des étrangers vivant en France, seraient actuellement concernées par cette situation. En 2011, un amendement (article 26) adopté par l'Assemblée nationale visant à restreindre les conditions d'accès au séjour pour raisons médicales a fait l'objet de polémiques. En effet, alors que le droit au séjour pour soins était jusqu'alors accordé à l'étranger gravement malade ne pouvant « effectivement bénéficier d'un traitement approprié dans son pays d'origine », ce critère d'accessibilité est désormais remplacé par celui de disponibilité. Un étranger gravement malade peut dès lors se voir refuser un droit au séjour si le traitement est « disponible » dans son pays, quand bien même son accès effectif ne serait pas assuré.

$11 \mathrm{La}$ circulaire DHOS/DSS/DGAS n ${ }^{\circ} 11$ du 16 mars 2005 définit les soins urgents de la manière suivante : "Soins dont l'absence mettrait en jeu le pronostic vital ou pourrait conduire à une altération grave et durable de l'état de santé de la personne ou de l'enfant à naître ». Cette circulaire est modifiée par celle du 7 janvier 2008 en ce qui concerne les ressortissants communautaires et les enfants mineurs ainsi que la facturation par les établissements de santé (cf. Bulletin Officiel $\mathrm{n}^{\circ} 2008-2$, http://www.sante.gouv.fr). 
contrôle policier qui s'ensuit sont parfois relevés, c'est surtout le décalage de temporalité entre la lourdeur administrative et l'urgence de la pose du diagnostic (Ménoret, 1999) qui est vivement dénoncé, surtout pour une maladie chronique, comme le cancer, susceptible d'évoluer très vite ${ }^{12}$.

Le contexte de méconnaissance (ou de faible connaissance) de la législation concernant les étrangers ajoute parfois une difficulté supplémentaire. Dans des établissements où le pourcentage de patients étrangers est très faible - et qui plus est relevant de l'AME $^{13}$-, les assistants sociaux ou le personnel des entrées ne sont pas nécessairement très au fait du droit des étrangers et certains relèvent la nécessité d'une formation ou soulignent l'importance de la formation récente qu'ils ont pu avoir à ce sujet.

L'éthique du soin dont se revendique l'équipe médicale tend alors à entrer en conflit avec la lourdeur des contraintes administratives ce qui peut conduire, dans des cas extrêmes et restant rares, à des pratiques à la limite de la légalité comme des hospitalisations non déclarées ou dont la déclaration est différée. Seuls quelques membres de l'équipe sont mis dans la confidence et la durée d'hospitalisation est réduite autant que possible, pour des questions de responsabilité, mais aussi de coût pour l'institution ${ }^{14}$. Ces tensions entre éthique humaniste du soin et légalité doivent en effet composer avec un troisième élément, une pression budgétaire de plus en plus forte dans les établissements de soin (Feroni, 2006). Ainsi, la combinaison de ressources structurelles défaillantes, ou du moins peu adaptées, et de la nécessité d'une prise en charge " fluide » de ces patients conduit les professionnels à des logiques de négociations constantes et de bricolage au cas par cas.

Pour les étrangers arrivés avec un visa touristique, le délai pour la mise en place d'une couverture médicale est également problématique puisqu'ils ne peuvent déposer une demande d'AME tant qu'ils ne sont pas en situation irrégulière. Leur statut ne leur permet pas non plus de bénéficier de la Couverture maladie universelle $(\mathrm{CMU})^{15}$ puisqu'ils ne sont pas résidents sur le territoire français et leur assurance au pays d'origine (assurance maladie et/ou assurance visa) - s'ils en ont une - ne couvre généralement pas les soins dispensés en France pour des pathologies telles que le cancer ${ }^{16}$. Certains en arrivent alors à « perdre » leurs papiers et, se trouvant ainsi en situation irrégulière, peuvent après justification de trois mois de présence sur le territoire, déposer une demande d'AME. Les quelques cas de ce type rencontrés correspondent à des étrangers ayant des réseaux de relations en France et qui ont pu se renseigner en détail sur les conditions requises et les démarches appropriées par le biais d'associations ou de services sociaux.

12 La rétroactivité de l'AME pose également parfois problème dans la mesure où elle prend effet à la date de réception du dossier complet. Or, des pièces complémentaires peuvent être demandées au compte-gouttes.

13 Cf. à ce sujet le rapport de IGAS/IGF (2007) sur l'aide médicale d'État.

$14 \mathrm{La}$ réduction des durées d'hospitalisation pour les personnes en situation irrégulière a également été relevée dans d'autres travaux, cf. Fassin et al. (2001).

15 Celle-ci a été mise en place en 2000 à destination des personnes ayant de très faibles ressources. Pour plus de précisions, cf. Maille et Toullier (2009).

16 Dans un bulletin daté de 2010, La ligue contre le Cancer à Paris rapporte également être de plus en plus confrontée à des étrangers atteints de cancer arrivés en France en qualité de touristes. 
Il n'est pas rare, en effet, que des étrangers saisissent l'occasion d'un séjour touristique pour effectuer un bilan de santé ou consulter un spécialiste, qu'il s'agisse de prévention ou que la cause de symptômes persistants n'ait pu être identifiée dans leur pays. Ces pratiques se retrouvent également parmi les travailleurs immigrés retraités engagés dans des allers-retours avec leur pays d'origine. Mais ces derniers, s'ils ont veillé aux procédures nécessaires, ont conservé leurs droits à l'assurance maladie et ne sont donc pas concernés par ces problèmes administratifs.

Parmi les étrangers arrivés en France en qualité de touristes, quelques-uns étaient conscients d'avoir un problème de santé. Pourtant, ils ne s'attendaient pas, le plus souvent, à devoir engager des traitements aussi lourds et coûteux qu'une radiothérapie ou une chimiothérapie, ce qui non seulement implique un imprévu financier, mais aussi toute une réorganisation familiale à envisager dans l'urgence, notamment pour ceux ayant des enfants au pays. Lorsque le cancer a été préalablement diagnostiqué dans le pays d'origine, des renseignements ont généralement été pris sur les possibilités de prise en charge. Leur famille, lorsqu'elle est présente en France, a pu s'adresser à son médecin généraliste, à des centres de soin, aux services hospitaliers ou à des associations de migrants. Parfois, elle présente, documents à l'appui, le protocole de soins programmé au pays et demande aux médecins si, ici, ils disposent d'un traitement plus efficace ${ }^{17}$. L'avis des professionnels consultés n'est pas toujours suivi, quand bien même ils répondent qu'ils n'ont pas de traitement alternatif à proposer. De plus, ces professionnels mettent en garde contre les difficultés voire l'impossibilité d'une prise en charge financière des soins par le système français. D'autres patients étrangers, notamment parmi ceux isolés familialement, peuvent arriver sans trop de connaissances précises.

Outre l'existence de liens familiaux, le choix de la Bretagne pour rechercher un traitement anticancéreux peut être orienté par les professionnels de santé dans les pays d'origine. Face aux limites de leurs prises en charge, certains ont pu conseiller à leur patient, ouvertement ou à demi-mot, d'aller « tenter leur chance en France ». Ils ont alors pu mentionner le nom d'un établissement dans lequel ils ont effectué un stage ou avec lequel ils ont été en contact dans le cadre d'échanges professionnels.

Certains étrangers arrivés avec un visa touristique ont tenté, après avoir été diagnostiqués, de déposer une demande de régularisation pour soins avant l'expiration de leurs visas, afin de bénéficier au plus vite, d'une couverture sociale. Les assistants sociaux, très sceptiques sur les chances d'aboutir de telles démarches, ne pensent pas nécessairement à les engager ou y renoncent d'emblée, persuadés que la préfecture invitera à renouveler la demande une fois le visa expiré ${ }^{18}$.

17 Les circulations migratoires autour du soin et plus généralement des actes de santé alimentent une mobilité internationale, comme cela a déjà pu être relevé dans d'autres contextes (Missaoui, 2010 ; Moullé et Duhamel, 2010).

18 En 2008, le cancer représente, au niveau national, 4 \% des pathologies invoquées pour une régularisation pour soin (première demande et renouvellement), derrière le $\mathrm{VIH}(18 \%)$, les problèmes psychiatriques $(16,7 \%)$, les hépatites $(8,2 \%)$, le diabète $(5,8 \%)$, la cardiologie $(5,5 \%)$, les problèmes locomoteurs $(4,7 \%)$. Cf. Thomas (2010). 
La situation de ces étrangers arrivant en France (avec ou sans papiers) dans un état de santé nécessitant des soins invite alors à débattre de la notion " d'immigration thérapeutique ». Guillou (2009) critique l'usage commun de cette notion à double titre. D'une part, celle-ci réduit la complexité des expériences migratoires et isole arbitrairement la variable santé. Comme le rappelle l'auteure, les causes des migrations sont plurielles et complexes. Non seulement elles se redéfinissent au fil de l'expérience migratoire, mais elles font l'objet d'une reconstruction narrative, en particulier ici à destination du chercheur. D'autre part, cette notion repose sur des soubassements politiques et idéologiques empreints de suspicion à l'égard des étrangers, suspectés de venir « profiter », voire « abuser » du système de soin national. Renversant la perspective, Guillou parle au contraire « d'immigration pathogène » pour rendre compte du fait que les immigrés attribuent souvent aux effets de l'émigration/immigration la cause de leur « mal ».

Certes, à travers un modèle physiopathologique de représentation de la maladie (Sarradon-Eck, 2004), le cancer est parfois attribué aux difficultés, stress et souffrances engendrés par leur condition d'étranger : "c c'est la France qui m’a donné le cancer », disent ainsi certains enquêtés ${ }^{19}$. Toutefois, pour d'autres, et notamment ceux nouvellement arrivés, la France est le pays qui, par contraste avec les systèmes de soins moins performants, défaillants ou clientélistes des pays d'origine ${ }^{20}$, offre un espoir de guérison ${ }^{21}$. Ces différentes représentations du pays d'immigration dans le rapport de cause à effet avec la maladie semblent ainsi être à moduler au regard, entre autres, des conditions et de l'expérience de la migration.

Il reste que venir en Bretagne pour recevoir des soins fait intervenir, comme précédemment évoqué, la combinaison d'un ensemble de dimensions (familiales, relations interpersonnelles, etc.) replaçant ces raisons de santé dans un cadre et une expérience migratoires plus larges, et nous rejoignons en cela l'analyse de Guillou (2009). En outre, par la suite, c'est autant l'évolution de la trajectoire de maladie (crainte de récidive ou récidive effective, poursuite des contrôles, traitements intensifs) que l'évolution de la situation migratoire et plus largement celle du parcours biographique (se construire ou reconstruire une situation professionnelle lorsque la durée des traitements en France conduit à la perte d'un emploi parfois haut placé au pays d'origine ; nouvelle mise en couple ; stigmatisation ou crainte de rejet de la part des proches au pays d'origine, notamment pour les femmes ne pouvant avoir d'enfants ; mise en place d'une procédure de regroupement familial ou non, etc.) qui poussent les intéressés à rester en France ou à repartir en tenant compte de la contrainte, à ce niveau également, des politiques d'immigration.

En ce sens, l'usage commun de la notion « d'immigration thérapeutique » biaise le regard. Car, même si la recherche d'un diagnostic et/ou d'un traitement peut orienter, combiné à d'autres enjeux, la venue en France, ces besoins de santé (ou quête de ressources) ne présupposent pas nécessairement, au départ, l'immigration et, de fait, se

19 De tels propos ont été recueillis auprès d'étrangers vivant en France depuis au moins quelques années. Ils ne rentrent donc pas dans la population d'étude dont il est fait mention dans le cadre de cet article.

20 Concernant l'Afrique de l'Ouest, cf. par exemple Jaffré et De Sardan (2003).

21 Le terme de guérison est souvent employé par les patients même si, d'un point de vue médical, c'est le terme de rémission qui s'applique en cancérologie du fait de l'incertitude pronostique. 
retrouvent d'emblée imbriqués dans une expérience sociale et migratoire beaucoup plus vaste.

\section{Le régime de « biolégitimité » versus le « corps persécuté »}

Si l'entrée dans le soin confronte les étrangers sans-papiers ou titulaires d'un visa touristique à divers obstacles administratifs, le cas des demandeurs d'asile peut aussi être complexe. Pour certains d'entre eux, le cancer a été diagnostiqué dans le pays d'origine et un premier traitement (opération, chimiothérapie, etc.) réalisé avant la migration a pu stabiliser leur état et/ou conduire à une rémission. D'autres découvrent leur cancer au cours de l'instruction de leur demande d'asile. Enfin, pour quelques étrangers atteints de cancer et arrivés en France clandestinement, demander l'asile peut être un moyen d'entrer dans les circuits d'aide et d'hébergement normalement conçus pour les demandeurs d'asile. Même si l'asile risque de leur être refusé, cela leur importe peu. Ce qui se joue pour eux, c'est l'obtention d'un hébergement et la mise en place d'une couverture maladie puisque les demandeurs d'asile bénéficient de la CMU durant l'instruction de leur dossier qui peut durer plusieurs années. D'autres saisissent l'occasion de la venue de proches demandant l'asile pour se « glisser» dans le circuit.

D'une manière générale, les professionnels intervenant dans les structures d'accueil et d'orientation des demandeurs d'asile soulignent l'état de santé de plus en plus dégradé dans lequel arrivent ces populations. Si celles-ci sont soumises à une visite médicale obligatoire lors de leur entrée en Centre d'accueil pour les demandeurs d'asile (CADA), la mise en place des soins est parfois un peu chaotique, notamment dans l'attente de l'ouverture de droits à la CMU. Un médecin intervenant dans un foyer pour demandeur d'asile regrette ainsi les retards dans le diagnostic du cancer d'une personne suivie :

«C'était un demandeur d'asile tchétchène avec un cancer ORL. Sa femme était déjà en France avec leur fils et lui est arrivé après. Et je me souviens très bien d'être à ma vacation du mardi après-midi au foyer, et il venait d'arriver, et je me suis dit: "Oh, lala, ce monsieur n'est vraiment pas très en forme!" ; ça se voyait vraiment. Du coup, je l'ai envoyé aux urgences tout de suite. J'ai trouvé qu'il y avait une mise en route qui était un peu lente. Parce que je l'ai envoyé aux urgences, et ils l'ont renvoyé au foyer. Ils avaient bien vu qu'il y avait quelque chose, mais ils $n$ 'avaient pas fait grand-chose de plus. Ils n'ont pas parlé de cancer, ils ont parlé de lésions, et ils l'ont renvoyé chez lui. Et c'est vraiment en ré-insistant, je l'ai envoyé en disant "non, il y a vraiment quelque chose, ça ne va pas" [...]. Il y a peut-être eu deux mois avant que ça soit vraiment pris sérieusement en charge, quoi, son cancer.

Peut-être qu'ils se sont dit : "là, de toute façon, on ne va rien pouvoir faire en urgence". Finalement, je l'ai renvoyé directement en consultation ORL, et là, l'ORL

l'a pris en charge » (entretien, juillet 2011).

Lorsque demande d'asile et problème de santé se conjuguent, la gestion des dossiers par les professionnels peut néanmoins diverger. Certes, aujourd'hui, en Bretagne comme plus généralement au niveau national, les demandes de régularisation au motif de la santé connaissent un taux d'acceptation supérieur aux demandes d'asile. En 2009 en France, $74 \%$ des demandes de régularisation pour soins ont reçu un avis favorable et le cancer représente $4,8 \%$ des pathologies invoquées pour ce motif (la région Bretagne 
a quant à elle rendu, en 2008, 2 \% du total des avis). En 2009 en Ille-et-Vilaine, le taux de réponses positives aux demandes de régularisation pour raisons médicales est de $72 \%$ (49\% des avis positifs le sont pour une durée supérieure à un an) et $2 \%$ des avis concernent le cancer (soit onze avis sur un total de 538) ${ }^{22}$. Par comparaison, en France en 2010, le taux global d'admission au titre de l'asile ${ }^{23}$ était de $27,5 \%$ et le taux d'accord délivré directement par l'OFPRA de 13,5\%. À l'échelle de la Bretagne, pour la même année, le taux d'admission des demandes d'asile par l'OFPRA était de 2,6 \% pour les Côtes-d'Armor, $5,2 \%$ pour le Finistère et de 16,9\% pour l'Ille-et-Vilaine (OFPRA, $2011: 82$ ).

Statistiquement, les régularisations pour soin ont donc plus de chance d'aboutir que les demandes d'asile, mais l'une et l'autre ne donnent pas accès aux mêmes droits. La régularisation pour soin s'accompagne d'une situation beaucoup plus précaire, dans la mesure où le titre de séjour est accordé pour quelques mois seulement. Son renouvellement n'est jamais garanti et prend fin dès lors que l'état de santé ne le justifie plus. De plus, selon qu'il s'agit d'une autorisation provisoire de séjour (APS) ou d'une carte de séjour temporaire (CST), l'étranger peut ou non être autorisé à travailler et bénéficier de prestations sociales comme l'allocation adulte handicapé ${ }^{24}$. Le statut de réfugié offre, comparativement, une plus grande stabilité juridique et ouvre plus de droits sociaux. Or, certains professionnels craignent qu'une demande parallèle de régularisation pour soin ne vienne délégitimer la procédure d'asile en cours. Cette appréhension est aussi partagée par certains demandeurs d'asile, arrivés en se sachant malades, mais qui disent ne pas avoir osé parler d'emblée de leur cancer (ou du moins de leurs symptômes) au risque d'attirer le soupçon sur la sincérité de leur demande.

Comme le souligne Mbaye (2009) à la suite de Fassin et al. (2001), en France, à partir de 1974 et de l'arrêt de l'appel à l'immigration de travail, la légitimité du corps malade s'est progressivement substituée à celle du " corps labeur " des travailleurs immigrés. Elle a aussi progressivement supplanté la légitimité des « corps persécutés » avec le durcissement de la politique d'asile au cours des décennies suivantes. Toutefois,

22 Dans les autres départements bretons, le nombre d'avis pour cancer en 2009 est encore plus faible : ils s'élèvent à neuf pour le Finistère, à six pour le Morbihan et à quatre pour les Côtesd'Armor. Nous n'avons pu obtenir le taux d'avis favorables à la régularisation pour raisons médicales dans ces départements. Les chiffres ici cités, obtenus en février 2012 auprès de la Direction générale de la santé, concernent les avis rendus par les médecins de l'ARS (Agence régionale de santé). Ils ne correspondent pas nécessairement aux titres de séjour effectivement délivrés par les préfectures, même si les données peuvent se corroborer. Il serait d'ailleurs intéressant de rechercher les éventuels écarts.

23 Somme des décisions d'accord de l'Office français de protection des réfugiés et des apatrides (OFPRA) et des décisions d'annulation de la Cour national du droit d'asile (CNDA).

24 Les préfectures délivrent de plus en plus des autorisations provisoires de séjour (APS) au lieu de cartes de séjour temporaires (CST). Les APS sont en théorie délivrées lorsque les intéressés ne peuvent justifier d'une résidence en France depuis au moins un an. Elles maintiennent les étrangers malades dans une situation sociale et juridique très précaire, car elles n'autorisent pas à travailler et leur durée de validité se limite à quelques mois (de trois à six mois généralement). De plus, elles rendent très difficile la sortie du territoire et ne donnent pas droit à l'Allocation Adulte Handicapé (AAH). À cela s'ajoute le fait que les bénéficiaires des APS rencontrent souvent, dans la pratique, des difficultés pour obtenir la CMU. Cf. Le Rapport de l'Observatoire du droit à la santé des étrangers (2008) La régularisation pour raison médicale en France, Un bilan de santé alarmant, [en ligne] consulté le 21 février 2012. URL : http//www.odse.eu.org 
ce « régime de bio-légitimité » (Fassin et al., 2001 ; Fassin, 2004) pourrait perdre de sa légitimité dès lors qu'il se conjugue à une requête d'asile. Un centre d'accueil pour demandeurs d'asile, en concertation avec un assistant social hospitalier dépassé par ce type de démarche, demande ainsi à une patiente d'Afrique centrale, atteinte de cancer et demandeuse d'asile, de « choisir » entre l'une ou l'autre de ces démarches.

D'autres professionnels, en revanche, n'hésitent pas à faire de suite une demande de régularisation pour soins, partant du constat que les demandes d'asile sont très souvent déboutées. Ces positionnements peuvent aussi relever de principes plus personnels dans la manière dont les professionnels se placent par rapport à la ligne officielle de partage entre réfugiés et migrants ${ }^{25}$.

Mais une autre dimension encore doit être prise en compte. Mettre d'emblée en œuvre, en cas de problèmes de santé, les démarches pour régularisation médicale est parfois une ligne de conduite impulsée par la direction de certaines structures d'aide aux demandeurs d'asile en vue de couper court à la logique du soupçon de certaines préfectures. Celles-ci, en effet, ont pu les accuser, plus ou moins explicitement, de demander un titre de séjour pour soin (injustifié) dès lors qu'une demande d'asile serait déboutée. Il s'agit alors, pour ces responsables, de montrer que ces deux dossiers (asile et santé) sont traités de front et de manière indépendante, sans être influencés par la suite administrative donnée à l'un ou à l'autre.

Qu'elles relèvent de logiques d'action individuelles ou collectives, ces pratiques donnent clairement à voir les effets des politiques d'immigration (et d'asile) sur les configurations d'entrée dans l'espace du soin.

\section{Pressions budgétaires croissantes : la figure de l'étranger qui « coûte cher »}

La combinaison du cadre des politiques d'immigration et du mode de fonctionnement des établissements conduit à un certain filtrage des patients. Entre les bureaux des entrées de différents établissements, des échanges téléphoniques peuvent avoir lieu lorsqu'un malade arrive d'un précédent centre de soin et que celui en passe de l'accueillir souhaite avoir des précisions sur des garanties financières lui paraissant confuses. Dans la plupart des établissements, les patients non couverts par la sécurité sociale sont censés déposer des chèques de garantie et payer une partie des soins par anticipation. Un devis accompagné d'un échéancier de paiement leur est présenté et ils doivent s'engager à l'honorer. Des employés d'un bureau des entrées relatent les pressions de plus en plus fortes « à récupérer l'argent» ou encore à faire "la chasse à la moindre recette » qui émanent de la comptabilité :

«L'année dernière, on a eu le cas d'un patient maghrébin venu pour se faire soigner, mais il est décédé en cours de traitement. Contre qui après voulez-vous vous retourner? La famille? Il avait accepté de payer à hauteur de 100 ou 200 euros par mois, puis il a été hospitalisé et ça a été interminable quoi! Il devait peut-être

25 Sur ce point, voir également les travaux d'Halluin (2008). 
L'image du patient étranger qui coûte cher au système de soin - et plus généralement à la société - est présente dans les représentations de certains soignants et administratifs, avec l'argument supplémentaire qu'en cancérologie, les molécules utilisées sont particulièrement onéreuses. Certains soulignent ainsi que la situation de la Bretagne les "protège relativement de l'immigration » et donc de patients potentiellement non payeurs. Un regard critique est également parfois posé sur les bénéficiaires du régime assistanciel de l'AME dans la mesure où, en l'absence de cotisation, ils tirent des avantages du système de solidarité nationale sans eux-mêmes y contribuer ${ }^{26}$. Comme le soulignent Guillou (2006) puis Hoyez (2011), ces représentations du « migrant-profiteur » voire « abuseur » du système de soins (et en sus, de la société) se construisent souvent à travers une mise en contraste des difficultés socio-économiques d'une part, et de ce qui est perçu comme une facilité d'accès aux soins d'autre part. Il en découle un débat sur l'équité, dans la mesure où «ce qui est donné à un étranger qui ne paie pas » (et qui donc ne le mérite pas) peut être perçu comme «quelque chose qu'on ne donne pas aux Français » (qui le méritent de par leur appartenance à la nation, alors même qu'ils peuvent également avoir des problèmes de sécurité sociale). Ces représentations, qui ne sont pas toutefois partagées par tous et révulsent même certains professionnels, s'ancrent au sein des établissements, dans un contexte institutionnel particulier.

En effet, qu'ils exercent au bureau des entrées, au service social ou dans des postes à responsabilité dans les unités de soin, les professionnels font part des pressions exercées par la direction dès lors qu'un patient non solvable a été pris en charge. Ce contexte est bien connu des « structures intermédiaires » (centres de soin gratuits, centres d'accueil des demandeurs d'asile, etc.) et leurs tactiques d'orientation des étrangers vers les établissements de santé en tiennent compte. Certaines, par exemple, vont préalablement appeler les urgences pour les avertir de l'arrivée d'un demandeur d'asile n'ayant pas encore de CMU. Mais, pour éviter des refus, d'autres optent pour mettre le personnel hospitalier devant le fait accompli, en y envoyant directement les patients ou, lorsque cela est possible, en les y accompagnant. Ces démarches, généralement réalisées par des travailleurs sociaux ou des bénévoles, misent sur la légitimité de leur statut ou du moins sur le regard témoin d'un « majoritaire » (Guillaumin, 2002) pour éviter les « refoulements » au guichet ${ }^{27}$.

Les relations interpersonnelles et les réseaux de connaissance entre médecins et spécialistes, hospitaliers ou de ville, jouent également sur l'orientation des patients. Tel médecin va adresser un étranger en situation précaire à tel confrère plutôt qu'à un autre, sachant que son collègue « compréhensif » fera tout pour amorcer une chimiothérapie ou une opération au plus vite, tandis que l'assistante sociale prendra en charge le dossier. Les praticiens peuvent aussi rechercher des solutions ad hoc en préférant, par exemple, la

26 La réforme de 2011, à travers l'institution d'un droit de timbre, produit toutefois un alignement sur un régime de type assurantiel.

27 Il arrive par ailleurs que des étrangers venus avec des visas touristiques s'adressent, en premier lieu, à des établissements privés (souvent pour des raisons de proximité spatiale avec leur lieu d'hébergement ou de rendez-vous plus rapidement obtenus) sachant que, dans tous les cas, les frais seront à leur charge. Mais, lorsqu'un cancer est diagnostiqué, les structures privées les réorientent quasi-systématiquement vers des établissements publics, ne voulant ou n'ayant pas les ressources nécessaires (en l'absence de service social) pour engager les démarches administratives qui s'imposent alors. 
programmation d'un traitement en ambulatoire à une hospitalisation plus onéreuse, même si l'état de santé du patient pourrait la justifier. Afin d'établir un devis plus facilement « acceptable » (par le patient comme par la direction), de telles orientations thérapeutiques peuvent être de prime abord privilégiées, quitte à ce qu'une hospitalisation soit envisagée plus tard si elle s'avère vraiment nécessaire. En s'inspirant de Lurie (1997), Lombrail, Pascal et Lang (2004 : 62) distinguent différents niveaux dans l'accès aux soins : à côté de l'accès primaire qui relève de " l'entrée dans le système ou son degré d'utilisation ", doit être pris en compte l'accès secondaire qui renvoie à la qualité dans le continuum des soins et à la manière dont ils sont administrés ou, autrement dit, à « la manière structurellement déterminée dont [les soins] se déroulent après un premier contact ». Au-delà de la distinction entre ces deux niveaux, c'est leur imbrication qu'il convient d'appréhender. Si Lombrail, Pascal et Lang (2004) montrent comment se cumulent difficultés d'accès primaire et secondaire, le mécanisme à l'œuvre peut être encore plus complexe. En effet, dans le cas précédemment rapporté, on a une différence de traitement ${ }^{28}$, pouvant être vue comme un « abaissement des normes de prises en charge » (Fassin et al., 2001 : 63), et qui est liée à des logiques institutionnelles dont les contraintes conduisent à une inégalité au niveau de l'accès secondaire (puisqu'un patient sans problème de couverture maladie aurait d'emblée été hospitalisé). Mais c'est justement cette inégalité dans l'accès secondaire qui permet l'accès primaire aux soins en contournant les obstacles administratifs.

Ces pratiques d'orientation, influencées par le poids des logiques administratives, sont sous-tendues par deux types de catégorisation. La première renvoie à une distinction entre patients solvables/non solvables; la seconde à la distinction entre patients avec papiers/sans papiers. Bien qu'elles puissent se recouper, ces deux types de catégorisation ne se superposent pas toujours puisqu'un patient en situation régulière peut ne pas avoir de couverture maladie (par exemple s'il possède un visa touristique) et qu'inversement, un patient en situation irrégulière peut avoir une sécurité sociale (AME). Il semble alors que, selon les situations et la position des professionnels dans l'espace du soin, l'un ou l'autre de ces types de catégorisation peut prendre le dessus dans les interactions. Ainsi, les responsables des services de soin (à différents niveaux de la hiérarchie, médecin, cadre supérieur, cadre) auront tendance à dire que le statut migratoire du patient leur importe peu et que c'est surtout leur non solvabilité qui peut poser problème en les contraignant, le cas échéant, à rendre des comptes à la direction. Pour les professionnels du service social (également comptables du volet financier devant la direction), la situation administrative du séjour est a contrario la dimension déterminante puisqu'elle va orienter le type de démarches à engager pour l'accès à une couverture maladie et donc permettre, par la suite, une solvabilisation.

La complexité des configurations d'entrée dans l'espace du soin met en relief les ambivalences entre droits aux soins, accès effectif aux soins et mise en œuvre des démarches de soin. Le droit d'accès aux soins ne garantit pas nécessairement une accessibilité facile en raison d'un ensemble de contraintes administratives et de l'existence de pratiques discriminatoires (lors du traitement des dossiers d'AME par exemple). Cette ambivalence se donne également à lire dans le décalage de temporalité entre l'urgence du soin et la lenteur des procédures administratives. Ce décalage fabrique des situations

28 Comme le rappelle Fassin et al. (2001), le terme de traitement désigne l'acte plutôt que l'intention. 
d'entre-deux où les démarches d'ouverture de droits à l'assurance maladie, alors qu'un cancer a été diagnostiqué, semblent bien longues à aboutir tant pour les professionnels que pour les patients. À cela s'ajoutent parfois, pour ces derniers, des difficultés relationnelles à dire les symptômes ou à être pris au sérieux. Il arrive en effet que leurs symptômes ${ }^{29}$ soient renvoyés à des troubles psychosomatiques liés aux maux de la migration au détriment d'examens complémentaires et, lorsque le cancer est enfin diagnostiqué, il est parfois déjà très évolué (Pian, 2011).

\section{LES TRAJECTOIRES DU MOURIR : GESTION AD HOC ET CONTRAINTES INSTITUTIONNELLES}

Les trajectoires du mourir sont un autre moment clé où se cristallise un ensemble de contraintes administratives et organisationnelles, imbriqué à la situation de migration et à des enjeux financiers divers. Ces contraintes interpellent voire bousculent la temporalité et la spatialité du soin. Dans le cas des étrangers atteints de cancer, le passage du curatif à l'entrée en soins palliatifs s'accompagne ainsi pour l'équipe médicale (comme pour le patient et ses proches si ceux-ci sont présents) d'une interrogation centrale : un retour au pays d'origine est-il souhaité ? À quel moment de la trajectoire du mourir ? Dans quelles conditions et comment le mettre en œuvre ?30

\section{Temporalité de l'annonce et retour au pays d'origine}

Les praticiens, notamment de soins palliatifs ou ayant en charge des cancers à très mauvais pronostics tels les cancers pulmonaires et ORL, insistent sur le fait que le suivi des patients nécessite toujours une perspective d'anticipation de la fin de vie, composant avec une des caractéristiques essentielles de la maladie cancéreuse, à savoir, son « incertitude temporelle " (Ménoret, 1999). Sans faire l'unanimité parmi les médecins et les cadres, certains pensent que cette anticipation demande à être accélérée dans le cas des personnes d'origine étrangère et ce, au regard de la problématique du retour au pays d'origine ${ }^{31}$.

Le mouvement des soins palliatifs, qui se développe en France dans les années 1970-1990, défend le droit au « bien mourir » en se démarquant d'une médecine jusque-là exclusivement destinée à guérir et dont les effets pervers, incarnés par « l'acharnement thérapeutique », sont progressivement dénoncés (Castra, 2003). L'un des principes

29 Dans le cadre des maladies chroniques en particulier, Baszanger (1986) parle du « long travail » du malade pour faire reconnaître la gravité de ses symptômes au fur et à mesure de leur aggravation, alors même qu'ils peuvent, dans un premier temps du moins, être minimisés par les médecins.

30 Les études portant sur les trajectoires du mourir des immigrés et étrangers en contexte de soin (Aggoun, 2006) sont peu nombreuses. En revanche, plusieurs travaux sur le vieillissement des immigrés en France abordent la problématique du retour au pays d'origine, du lieu de funérailles et du rapport à la mort. Voir par exemple Attias-Donfut (2006).

31 Parmi ceux qui ne soutiennent pas cette position, certains estiment qu'ils n'ont ni à aborder ni à faire advenir le sujet si la demande ne vient pas du patient (ou de ses proches). Une telle approche leur semble en effet « déplacée» : «ce n'est pas à moi de dire s'il faut qu'il meure ici ou là-bas. S'il est ici, c'est qu'il a des raisons d'être ici et ce n'est pas à moi de demander s'il veut rentrer chez lui », souligne ainsi un professionnel de santé. 
éthiques des soins palliatifs est de prendre en compte le processus d'acceptation de la mort (Rimbert, 2007) et les équipes qui s'en réclament sont particulièrement attentives à l'évolution psychologique des patients en stade terminal ${ }^{32}$. Or, certains médecins expliquent que l'enjeu du retour au pays les contraint parfois à " brusquer » les annonces de fin de vie, quitte « à faire violence au patient » afin que celui-ci prenne rapidement la décision de rentrer ou non avant qu'il ne soit trop tard ${ }^{33}$. L'incertitude temporelle de la « trajectoire descendante » (Ménoret, 1999) du cancer se double en effet d'un éclatement spatial entre ici et là-bas qui vient en complexifier la gestion. Dans l'idéal des soignants, le retour-s'il doit avoir lieu - doit s'effectuer avant que l'état de santé ne soit trop dégradé. D'une part, pour permettre au patient de «profiter» de ses derniers moments de vie au pays et que cela ne soit pas trop dur pour les proches qui le voient revenir gravement malade, d'autre part, pour des raisons logistiques afin que l'intéressé puisse voyager en avion sans être alité et qu'un accompagnement médicalisé ne soit impératif. Car, à trop attendre, le retour risque de nécessiter un rapatriement sanitaire, très coûteux et difficile à mettre en œuvre. En Bretagne en outre, dans un contexte où les patients cancéreux d'origine étrangère sont proportionnellement peu nombreux, les équipes sont peu habituées à gérer ce type de démarches. Et, même dans des régions à plus forte immigration où les situations de rapatriements sanitaires peuvent être plus fréquentes, ces derniers sont toujours difficiles à mettre en place (Atmani, 2004 ; Marin, 2004 ; 2007). L'exemple suivant illustre les difficultés d'organisation que soulèvent de tels retours en fin de $v^{34}{ }^{34}$ ainsi que la défaillance des ressources structurelles nécessaires.

Madame Y. est une patiente originaire des Balkans, âgée de soixante-dix ans, atteinte d'un cancer du poumon. Parlant très peu français, coiffeuse de métier, elle est arrivée il y a quelques années en France pour aider son fils et sa belle-fille à garder leurs enfants. Les autres membres de sa famille, dont ses filles et fils, sont dans les Balkans ou dans d'autres pays étrangers. En 2007, elle est hospitalisée et subit une chimiothérapie puis une radiothérapie. Face à l'échec thérapeutique, elle est transférée dans un service de soin palliatif. Son fils ne veut pas que sa mère soit mise au courant du pronostic, et souhaite organiser, en accord avec celle-ci, son retour au pays. Il en informe l'équipe dès l'entrée dans le service. Une semaine plus tard, le service social contacte le service des étrangers de la mairie afin de se renseigner sur les procédures du rapatriement sanitaire, la patiente n'étant pas en mesure de prendre l'avion sans « soutien » médical. Trois jours plus tard, le service reçoit une réponse de la mairie qui, elle-même, est en attente d'une réponse de l'ANAEM (Agence nationale de l'accueil des étrangers et des migrants, remplacée en 2009 par l'Office français de l'immigration et de l'intégration). La personne relais à l'ANAEM met toutefois en doute la possibilité de réalisation de la demande, même si elle transmet le dossier au siège à Paris. Deux jours plus tard, la réponse qui revient de l'ANAEM est négative. Le lendemain, l'équipe propose un rendez-vous à la famille (d'autres enfants sont arrivés de l'étranger) pour le jour suivant. Lors de cette rencontre,

32 Sur les modèles psychologiques du deuil incorporant une modélisation normative du « bien mourir », cf. Marche (2009: 33-37).

33 Ces prises de décisions engagent également, le cas échéant, des négociations familiales - parfois complexes - entre les proches ici et là-bas.

34 Des retours " non définitifs » du vivant, mais considérés comme des adieux à adresser aux proches avant de revenir mourir en France sont aussi parfois envisagés. Ils posent alors la question du « bon moment» au regard de l'état de santé. 
les proches sont informés de l'impossibilité de la prise en charge du rapatriement sanitaire dont le coût s'élève à plus de 45000 euros. Le service social s'engage à contacter le service social de secteur pour voir si certaines aides peuvent être trouvées par d'autres biais. La famille est également appelée à se mobiliser pour trouver des financements. Elle prend notamment contact avec un imam pour tenter d'avoir le soutien de la communauté musulmane. Une semaine plus tard, la patiente décède dans le service, soit trois semaines environ après son entrée (archive, dossier patient, consulté en juillet 2011).

Pour les étrangers sans-papiers, les démarches du retour - par rapatriement sanitaire ou non - sont encore plus complexes et le décalage entre le rythme institutionnel et la temporalité de l'urgence est d'autant plus prégnant ${ }^{35}$. Le rythme de l'urgence se cale sur l'état physiologique du patient qui peut se dégrader très vite, ce qui implique donc de devancer le décès. Le rythme institutionnel est celui de l'administration où il faut compter avec les attentes, les relances pour obtenir le document demandé (sauf-conduit, autorisation à quitter le territoire), être mis en relation avec la bonne administration et au sein de celle-ci, le bon interlocuteur, ou encore recevoir les autorisations sanitaires nécessaires.

\section{Assurer une transition médicale entre ici et là-bas}

Outre l'organisation du voyage, c'est le suivi médical à distance qui doit composer avec un cadre institutionnel rigide et peu adapté. Lorsqu'un retour en fin de vie est mis en place, les praticiens cherchent à établir des contacts avec un établissement de santé dans le pays d'origine, afin de s'assurer des soins qui pourront être dispensés en cas de besoin, même si le maintien à domicile est d'abord envisagé. En effet, les médecins se sentent investis du devoir d'assurer la «transition » et ces retours, qu'ils aboutissent ou non d'ailleurs, génèrent parfois un sentiment d'inaccompli parmi les équipes qui regrettent de ne pas avoir pu « accompagner » le patient jusqu'au bout. Pour faire le lien avec l'univers médical là-bas, les professionnels - parfois par le biais du service social - font feu de tout bois dans une logique relevant de la « débrouille». Ayant souvent une faible connaissance des pays d'origine, ils font jouer leurs réseaux personnels et d'interconnaissances pour obtenir qui le nom d'un hôpital, qui le nom d'un praticien. Ces ressources sociospatiales sont puisées dans le monde médical ou non : certains s'adressent à des amis ou des confrères originaires du même pays que leur patient ou exerçant dans des services à plus forte concentration de population étrangère, comme les services de VIH ou de lutte contre la tuberculose ; d'autres se tournent vers des collègues travaillant dans des régions à plus forte immigration, comme en Île-de-France, ou encore vers des collègues connus pour avoir suivi une formation sur l'interculturalité, etc. Des informations peuvent également être recueillies à partir de sites Internet, comme le forum de la société française

35 Le décès des étrangers en France - qu'un retour au pays ait été souhaité ou non du vivant - est confronté soit au coût du rapatriement du corps, soit au coût des obsèques. La solidarité communautaire, associative et confessionnelle est alors souvent mobilisée pour recueillir des fonds. Toutefois, les frais à engager pour un rapatriement du corps demeurent élevés et s'y ajoutent des démarches administratives plus ou moins complexes. Si l'inhumation peut être une solution, elle est contraire à certaines confessions comme pour la religion musulmane. Il reste que les étrangers vivant depuis plusieurs années en France, notamment parmi les travailleurs immigrés retraités ou non - souscrivent très souvent à une assurance rapatriement en cas de décès, ce qui est beaucoup moins fréquent chez les étrangers nouvellement arrivés. 
des accompagnants en soins palliatifs dont l'accès est réservé aux adhérents. Un professionnel témoigne ainsi :

«On voit de temps en temps des messages qui disent: "on accueille actuellement dans notre service un patient d'origine étrangère, on aimerait organiser un retour, est-ce que vous connaissez un hôpital sur place, dans un secteur géographique pour accueillir un patient en fin de vie, pour mettre en place un traitement, de la morphine..."”.

Enfin, pour les étrangers qui ne sont pas isolés familialement, les proches jouent un rôle essentiel dans la mise en contact avec le milieu médical dans le pays d'origine. Ils se mobilisent, par exemple, pour rechercher un établissement voire un médecin si possible réputé, adressent des demandes de devis ou encore se renseignent sur l'accessibilité et la disponibilité de tel ou tel traitement.

\section{Circulations transnationales des médicaments et prise en charge palliative}

À côté de la question du lien entre établissements ici et là-bas, l'organisation du retour soulève le problème de la prise en charge médicamenteuse. Certains traitements ou antalgiques, tels que la morphine, dont bénéficient les patients en fin de vie en France, et qui participent de la «modélisation de la bonne mort» sans souffrance (Atmani, 2004), ne sont pas disponibles ou accessibles dans un certain nombre de pays dits « en voie de développement » ou " sous-développés » : soit ils n’existent pas sur le marché (du moins le marché officiel), soit ils sont très onéreux alors que les intéressés ne jouissent pas, le plus souvent, d'une sécurité sociale dans leur pays. Ces contraintes institutionnelles donnent lieu à une circulation transnationale des médicaments et contraignent à des pratiques à la limite de la légalité, dont l'objectif affiché est d'assurer, autant que possible, le bien-être du patient. Prescriptions supérieures aux doses à administrer afin de prolonger la durée du traitement ; récupération de médicaments lorsque ce système existait encore, permettant de partir avec un plus grand approvisionnement; ou encore médiation de proches en France qui se chargent de venir chercher le renouvellement des ordonnances puis d'envoyer les médicaments au pays par voie postale ou par un intermédiaire ${ }^{36}$. Ces circulations migratoires (de Tapia, 2005 ; Guillon et Stockman, 2000) ${ }^{37}$ de médicaments, qui n'échappent pas au risque de rupture de la chaîne, ne sont pas propres à la fin de vie en oncologie. Elles concernent potentiellement tous les traitements difficilement accessibles ou inexistants dans les pays d'origine, à l'instar de ce qu'a pu, par exemple, observer Bascougnano (2009) au sujet du VIH-Sida.

36 Comme le rappelle Marin (2007), une circulaire régit la prescription des traitements pendant un séjour à l'étranger. Mais, selon le régime d'affiliation du patient (AME, CMU, Sécurité sociale) et le type de traitements, les modalités sont différentes. Les conditions sont d'autant plus strictes pour les médicaments relevant de la famille des stupéfiants, comme la morphine.

$37 \mathrm{Tel}$ que l'utilisent les auteurs, ce concept ne se limite pas à rendre compte de la mobilité humaine (sous forme de va-et-vient, d'aller-retour, etc.), mais englobe plus largement les relations denses et continues (économiques, sociales, idéelles, matérielles, financières, etc.) que ces derniers tissent entre un ou plusieurs espaces géographiques. 
Ces pratiques ad hoc, relevant de la débrouille dans un système inadapté à ce type de situation, ne vont pas sans soulever certaines craintes et interrogations parmi les professionnels ${ }^{38}$ : comment s'assurer de l'usage effectif de ces médicaments dans les pays d'origine ? Ne risquent-ils pas d'être dérobés ? Des complications ne risquent-elles pas de survenir lors du passage à la douane, surtout si le voyage en avion implique une escale dans un pays tiers? Néanmoins, malgré les doutes qui agitent de nombreux praticiens, l'éthique $\mathrm{du}$ « bien mourir » les conforte dans ces recours par défaut. De plus, des considérations d'ordre gestionnaires et financières peuvent leur permettre, intérieurement du moins, de justifier et légitimer leur choix vis-à-vis de l'institution et de la société : car, quoi qu'il en soit, ces prescriptions à la limite de la légalité coûtent moins cher au système de soin qu'une hospitalisation et ce d'autant plus lorsque le patient risque de laisser derrière lui des impayés. Un personnel du bureau des entrées s'exprimait ainsi à propos d'un patient maghrébin « isolé » :

«Avec l'assistant social, on a tout fait pour l'inciter à partir, à rentrer chez lui, car il n'avait pas de couverture. On ne voulait pas qu'il décède ici, car ça allait encore augmenter la facture, mais finalement c'est ce qui s'est passé quand même »

(journal de terrain, avril 2011).

Si les équipes restent tournées vers le soin, les pressions budgétaires demeurent donc fortement présentes. Dans un contexte de réduction des durées d'hospitalisation (Ménoret, 1999) motivée par la maîtrise des dépenses de santé, les services sont soumis à une politique du chiffre les incitant à ne pas dépasser une durée moyenne de séjour. Devoir garder un patient « trop longtemps » peut devenir générateur de tensions, surtout lorsque viennent s'ajouter des difficultés d'ordre relationnelles et communicationnelles et/ou que le nombre limité de lits nécessite une gestion à flux tendus. Un assistant social témoigne en ce sens d'échanges équivoques avec un cadre à propos de Madame R., la patiente géorgienne dont il a été question plus haut :

« Le cadre me disait : - Non, ça se serait bien qu'elle rentre, hein. C'est quand même mieux de mourir dans son pays.

- Oui, peut-être, mais est-ce qu'elle, elle le souhaite? - Oui, oui, elle a pu le dire, et puis là, tu te rends compte, ça fait plus de 160 jours qu'elle est dans le service, ça n'est plus possible. Moi, la direction me demande de mettre un terme à certaines hospitalisations ».

Ici, l'argument de l'altérité culturelle (lié au « mourir chez soi », mais aussi plus largement à l'évocation de relations difficiles avec la patiente en raison de la distance culturelle et de comportements culturellement excessifs) ${ }^{39}$ vient conforter, voire nourrir,

38 Celles-ci peuvent également être partagées par les patients.

39 Bien qu'il ne soit pas possible ici de développer ce point, les patients originaires des pays de l'ex-bloc communiste sont catégorisés comme particulièrement difficiles, exigeants, voire agressifs, par de nombreux soignants (cf. également Guillou, 2006). Dans une logique globalisante et essentialisante, leur comportement tend à être étiqueté sous l'enseigne du « syndrome méditerranéen » se caractérisant par une exagération de la douleur et des symptômes. Comme le dit un professionnel, ces patients sont « culturellement bruyants face à la maladie » puis il ajoute " ou du moins ils font beaucoup de bruit dans les services ». 
l'argument institutionnel mis en en avant par le cadre. Le contexte institutionnel impose aux équipes des contraintes auxquelles elles tentent de s'ajuster, même si cela peut conduire à des "gestions parfois ambiguës ». Certes, les pressions aux sorties d'hospitalisations concernent également des patients qui ne sont pas d'origine étrangère, mais les enjeux prennent une tout autre ampleur lorsque l'alternative se joue dans un retour au pays d'origine. Car, si le va-et-vient entre hospitalisation à domicile et centre de soin peut être relativement fluide lorsque la domiciliation est circonscrite dans un certain périmètre géographique et que les conditions sociales requises (Boada, 2007) sont réunies, ces allerretour deviennent impossibles à une échelle internationale et plus encore à ce stade de la maladie. De surcroît, l'orientation extérieure vers d'autres établissements est, pour toutes les raisons déclinées précédemment, souvent plus difficile à mettre en œuvre que pour d'autres patients.

\section{CONCLUSION}

L'espace du soin et les trajectoires de maladie qui y prennent place peuvent être appréhendés au prisme des trois dimensions - de masse, de durée et de contexte - qui, pour suivre Grossetti (2011), sont constitutives de l'espace des phénomènes sociaux ${ }^{40}$. La prise en compte de la combinaison de ces trois dimensions, qui se déclinent chacune sur différentes échelles, offre un cadre méthodologique et analytique pertinent pour rendre compte des contraintes administratives auxquelles se trouvent confrontés les étrangers atteints de cancer ainsi que les répertoires de pratiques (profanes et professionnelles) mises en œuvre pour y répondre. En effet, la gestion, au sens de Strauss, des trajectoires de maladie (et du mourir) fait l'objet d'interactions et de négociations in situ entre patients et professionnels, mais aussi entre structures et réseaux d'acteurs jouant un rôle de " pont » avec les établissements de soin. Ces pratiques, observables sur le terrain (en tant qu'échelle d'observation et niveau d'action), composent avec le cadre des politiques d'immigration et de santé qui instaure un marquage réglementaire et législatif vis-à-vis duquel les acteurs sont amenés à se positionner, et qu'il est donc indispensable de prendre en compte en termes d'échelle d'analyse (traitement des données). Concernant les politiques d'immigration, l'on retiendra les législations actuelles restreignant le droit au séjour des étrangers ainsi que, à la jonction des politiques de santé, les réformes relatives à l'accès aux soins, au séjour et à la couverture maladie des étrangers gravement malades. Mais ce sont aussi les réformes plus générales du système de soin et notamment celles concernant les gestions organisationnelles et budgétaires des établissements de soin qui pèsent lourdement sur les trajectoires de maladie ici évoquées.

Ainsi replacée, la focale adoptée ne doit pas toutefois prêter à confusion : si l'accent a été mis sur ce qui " pose problème » à deux extrémités de la trajectoire de maladie - l'entrée dans le soin et le temps du mourir -, tous les cas de prises en charge ne sont pas aussi complexes. Il n'en reste pas moins que ces derniers sont révélateurs

40 Dans le modèle proposé par l'auteur pour dépasser l'opposition macro-micro tout en la prenant en compte, la masse fait référence au nombre d'acteurs impliqués qui peuvent être différentes entités (interactions, organisations, États, etc.) ; la durée fait référence à la temporalité des phénomènes sociaux ; enfin, le contexte réfère aux différents " mondes sociaux » impliqués. Les contextes peuvent être définis à différents niveaux de masse et être eux-mêmes des « entités agissantes ». 
d'un certain nombre d'enjeux : révélateurs, tout d'abord, des limites d'un système de soin et de ses effets pervers qui peuvent contribuer à des inégalités " par omission » et par « construction » (Lombrail, Pascal et Lang, 2004) ${ }^{41}$; révélateurs, ensuite, de l'entredeux dans lequel se trouvent les équipes, entre une éthique du soin " humaniste » et un ensemble de contraintes institutionnelles qui soulèvent des enjeux sociaux et moraux. Les propos suivants, tenus par un praticien, résument la mise en tension quasi " schizophrène " (Fassin, 2007) ${ }^{42}$ qui travaille l'éthique, le politique et la politique : "C'est vrai qu'on ne peut pas accueillir toute la misère du monde. Mais quand ils sont là, on se retrouve devant le fait accompli. On les prend en charge » souligne ce médecin. Comme le souligne Agier (2008), la question politique ne se réduit pas aux idéologies qui se « cachent toujours plus ou moins "derrière" ou "au cœur" des interventions de contrôle, d'assistance ou de soin, ce en quoi leur sens serait in fine politique » : elle relève également de la politique, au sens des actions, des prises de position, des conflits qui « fondent ou refondent [...] en situation l'existence du sujet ». En ce sens, cet article montre comment la prise en charge d'étrangers précaires place les professionnels de santé au cœur d'enjeux doublement politiques qui bousculent à la fois des normes et des pratiques et met en scène une catégorisation des patients selon deux types de critères : papiers/sans papiers ; solvables/non solvables. Or, ces enjeux sont tout aussi prégnants dans les configurations d'entrée dans l'espace du soin que dans les «trajectoires du mourir ». Cet article pointe également, dans des contextes locaux jusque-là relativement peu confrontés à ces profils de patients, les différents types de ressources (structurelles et interpersonnelles), sur lesquelles les professionnels s'appuient ou qu'ils peuvent mobiliser, et leurs limites. Enfin, il éclaire les logiques de mise en forme des trajectoires de maladie par les patients eux-mêmes (notamment au niveau de l'entrée dans l'espace du soin), de manière étroitement imbriquée à la situation et à l'expérience migratoires.

Nous avons ainsi cherché à contribuer tant aux réflexions menées par la sociologie des migrations que par l'anthropologie et la sociologie de la santé. En mettant à jour les effets des politiques d'immigration et de santé sur les trajectoires de maladie et du mourir, nous rejoignons des problématiques clés de la sociologie des migrations s’intéressant à la "santé des migrants », tout en mobilisant des données empiriques relativement nouvelles. D'une part, les travaux qualitatifs et constructivistes qui portent sur le cancer des étrangers (et notamment sur les primo-arrivants) sont peu développés ; d'autre part, la question du retour en fin de vie des étrangers hospitalisés constitue un champ d'étude encore en friche. En s'intéressant à l'imbrication entre contexte de migrations internationales et trajectoires de maladie, cet article participe des réflexions qui se développent en anthropologie autour des questions de santé, de mobilité et de globalisation (Sakoyan,

41 Pour les auteurs, les inégalités par omission résultent d'inégalités produites par défaut dans le cadre du fonctionnement routinier du système de soins, pas ou peu adapté à telles ou telles situations (par exemple ici le lien médical avec le pays d'origine en cas de retour en fin de vie); les inégalités par construction renvoient à « des modalités programmées de fonctionnement » du système de soin (par exemple les difficultés administratives d'accès aux soins pour certaines catégories d'étrangers).

42 Fassin parle d'une « schizophrénie politique » entre d'une part, des politiques d'immigration qui, en devenant de plus en plus restrictives et répressives, renforcent, voire alimentent, la précarité des migrants et d'autre part, une politique de santé qui, revendiquant les " principes généraux de la solidarité et de l'égalité, étend le dispositif de protection sociale aux plus vulnérables ». 
Musso et Mulot, 2011). Mais il rejoint aussi des préoccupations plus « classiques » de la sociologie de la santé à propos du « travail» de gestion des trajectoires, des ressources et logiques (profanes, professionnelles, structurelles) qui y sont associées ainsi que de l'impact du contexte institutionnel sur les pratiques des acteurs. In fine, ce croisement sous-disciplinaire invite à élargir la réflexion aux circulations dans l'espace du soin tout au long de la trajectoire de maladie, dans l'ensemble de sa temporalité, tout en prenant en compte les différentes échelles spatiales impliquées.

\section{Références bibliographiques}

AEBERHARD Patrick et LEBAS Jacques (Éds.) (2011) L'accès aux soins des migrants, des sanspapiers et des personnes précaires : pour un droit universel aux soins, Bordeaux, Études hospitalières, $106 \mathrm{p}$.

AGGOUN Atmane (Éd.) (2006) Les musulmans face à la mort en France, Paris, Vuibert, 156 p.

AGIER Michel (2008) Gérer les indésirables. Des camps de réfugiés au gouvernement humanitaire, Paris, Flammarion, $349 \mathrm{p}$.

ATMANI Isabelle (2004) De la bonne mort au bon mort, in Simone Pennec Éd., Des vivants et des morts. Des constructions de la «bonne mort », Brest, Université de Bretagne Occidentale, CRBC/ARS, pp. 131-142.

ATTIAS-DONFUT Claudine (2006) L'enracinement. Enquête sur le vieillissement des immigrés en France, Paris, Armand Colin, 357 p.

BASCOUGNANO Sandra (2009) Savoirs, idéologies et traitements différentiels discriminants dans les rapports soignants/soignés. La prise en charge hospitalière du VIH-sida à la Réunion et en Ille-de-France, Thèse de doctorat de sociologie, Université Paris 7.

BASZANGER Isabelle (1986) Les maladies chroniques et leur ordre négocié, Revue française de sociologie, 27 (3), pp. 3-25.

BERCHET Caroline et JUSOT Florence (2012) État de santé et recours aux soins des immigrés en France : une revue de la littérature, Bulletin épidémiologique hebdomadaire, 2-3-4, pp. 17-21.

BOADA Hélène (2007) La prise en charge au domicile est-elle toujours possible en Seine SaintDenis ?, Liaison ASP, 36, pp. 18-19.

BOUJASSON Laura (2009) De l'épreuve du travail à l'épreuve de la reconnaissance du cancer en maladie professionnelle, Mouvements, 58 (2), pp. 56-65.

BOUCHARDY Christine, PARKIN Donald M. and KHLAT Myriam (1994) Cancer mortality among Chinese and South-east Asian migrants in France, International Journal of Cancer, 58 (5), pp. 638-643.

BOUCHARDY Christine, PARKIN Donald M., WANNER Philippe et KHLAT Myriam (1995) Cancer mortality among Sub Saharan African migrants in France, Cancer, Causes and Control, 6 (6), pp. 539-544.

BOUCHARDY Christine, PARKIN Donald M., WANNER Philippe et KHALT Myriam (1996) Cancer mortality among north African migrants in France, International Journal Of Epidemiology, 25 (1), pp. 5-13.

BRAHIMI Michèle (1980) La mortalité des étrangers en France, Population, 3, pp. 603-632.

CASTRA Michel (2003) Bien mourir. Sociologie des soins palliatifs, Paris, Puf, 365 p.

CHAOUCHI Stéphanie, CASU Catherine et LEDESERT Bernard (Éds.) (2006) État de santé et accès aux soins des migrants en France. Analyse et synthèse bibliographique, Rapport de l'Observatoire régional de la santé, Languedoc-Roussillon, consulté le 17 mars 2011 [en ligne]. URL : http://www.ors-lr.org/travauxetetudes/pdf/Rapportmigrants.pdf 
COGNET Marguerite, BASCOUGNANO Sandra et ADAM-VÉZINA Émilie (2009) Traitement différentiel dans les parcours thérapeutiques. Maladies infectieuses : Vih-Sida, tuberculose, Rapport final, Urmis-Soliis.

COGNET Marguerite, GABARRO Céline et ADAM- VÉZINA Émilie (2009), Entre droit aux soins et qualité des soins, Hommes et migrations, 1282, pp. 54-65.

CURTIS Sarah, GESLER Wilbert M., PRIEBE Stefan and FRANCIS Susan (2009) New spaces of inpatient care for people with mental illness: a complex "rebirth" of the clinic?, Health and Place, 15, pp. 340-348.

DARMON Nicole and KHLAT Myriam (2001) An overview of the health status of migrants in France in relation to their dietary practices, Public Health Nutrition, 2 (4) pp. 163-172.

DUSSUD François-Xavier, LE ROHO Yves, LEVY David, PORTIER Jean-François et ROUXEL Michel (2004) Atlas des immigrés en Bretagne, INSEE/FASILD, consulté le 21février [en ligne]. URL : 2012http://www.insee.fr

DYCK Isabel and O'BRIEN Patti (2003) Thinking about environment: incorporating geographies of disability into rehabilitation science, Canadian geographer, 47 (4), pp. 400-413.

FASSIN Didier (2007) La santé des étrangers : une question politique, La santé de l'Homme, 392, pp. 15-17.

FASSIN Didier (2004) Le corps exposé. Essai d'économie morale de l'illégitimité, in Didier Fassin et Dominique Memmi Éds., Le gouvernement des corps, Paris, EHESS, pp. 237-268.

FASSIN Didier (1991) L'espace politique de la santé. Essai de généalogie, Paris, Puf, 324 p.

FASSIN Didier, CARDE Estelle, FERRE Nathalie et MUSSO-DIMITRIJEVIC Sandrine (2001) Un traitement inégal. Les discriminations dans l'accès aux soins, Rapport de recherche, CRESP, $270 \mathrm{p}$.

FERONI Isabelle (2006) Évolutions institutionnelles, transformations de l'activité : le contexte des restructurations hospitalières des années quatre-vingt-dix (Commentaire), Sciences sociales et santé, 24 (4), pp. 129-133.

GRILLO Francesca, SOLER Marion et CHAUVIN Pierre (2012) L'absence de dépistage du col de l'utérus en fonction des caractéristiques migratoires chez les femmes de l'agglomération parisienne en 2010, Bulletin épidémiologique hebdomadaire, 2-3-4, pp. 45-47.

GROSSETTI Michel (2011) L'espace à trois dimensions des phénomènes sociaux. Échelles d'action et d'analyse, SociologieS, Expériences de recherche, Régimes d'explication en sociologie, consulté le 14 février 2012 [en ligne]. URL : http://sociologies.revues.org/3466

GUILLAUMIN Colette (2002) L'idéologie raciste, Paris, Gallimard, 378 p.

GUILLON Michèle et SZTOKMAN Nicole (Éds.) (2000) Géographie mondiale de la population, Paris, Ellipses, 319 p.

GUILLOU Anne (2006) Patients immigrés dans la région rennaise. Le regard des médecins généralistes, Rapport d'enquête, Réseau Ville-Hôpital-Rennes, Odris, Addras, Fasild, 49 p., consulté le 21/02/2012 [en ligne]. URL : http://www.odris.fr/documents/etudes/RVH_SM2_ RapportFINAL.pdf

GUILLOU Anne (2009) Immigration thérapeutique, immigration pathogène. Abandonner le " parcours thérapeutique » pour « l'expérience migratoire », Sciences sociales et santé, 27 (1), pp. 63-71.

HACHIMI-ALAOUI Myriam et NACU Alexandra (2010) Soigner les étrangers en situation irrégulière - Des politiques migratoires aux postures professionnelles, Hommes et migrations, 1284, pp. 163-173.

HALLUIN Estelle d' (2008) Les épreuves de l'asile. De la politique du soupçon à la reconnaissance des réfugiés, Thèse de doctorat de sociologie, Paris, EHESS.

HOYEZ Anne-Cécile (2011) L'accès aux soins des migrants en France et la « culture de l'initiative locale ». Une analyse des contextes locaux à l'épreuve des orientations nationales, Cybergeo: European Journal of Geography, Espace, Société, Territoire, 566, consulté le 25 janvier 2012 [en ligne]. URL : http://cybergeo.revues.org/24796 
IBRAHIMA Saïd (2009) Comment la culture surdétermine la souffrance des malades du cancer, in Patrick Ben Soussan et Éric Dudoit Éds., Les souffrances psychologiques des malades du cancer, Paris, Springer, pp. 131-136.

IGAS/IGF (2007) Rapport sur la gestion de l'aide médicale d'État, consulté le 2 févier 2012 [en ligne]. URL : http://www.ladocumentationfrancaise.fr/rapports-publics/074000345/index.shtml

JAFFRE Yannick et OLIVIER DE SARDAN Jean-Pierre (Éds.) (2003) Une médecine inhospitalière. Les difficiles relations entre soignants et soignés dans cinq capitales d'Afrique de l'Ouest, Paris, Karthala, 449 p.

KHLAT Myriam, SERMET Catherine et LAURIER Dominique (1998) La morbidité dans les ménages originaires du Maghreb, sur la base de l'enquête Santé de l'Insee 1991-1992, Population, 6, pp. 1155-1184.

KONE Tiédaba (2010) Cancer, culture et soins : une approche humaniste de la prise en charge des patients, Paris, L'Harmattan, $200 \mathrm{p}$.

LANNA Flavienne, MARCHAND Anne et THEBAUD-MONY Annie (2000) Cancer et travail. Des liens et des droits niés, Plein Droit, 86, pp. 1-20.

LOMBRAIL Pierre, PASCAL Jean et LANG Thierry (2004) Accès au système de soins et inégalités sociales de santé : que sait-on de l'accès secondaire, Santé, société et solidarité, 2, pp. 67-71.

LURIE Nicole (2007) Studying access to care in managed care environments, Health Services Research, 32, pp. 691-701.

MAILLE Didier et TOULLIER Adeline (2009) Les dix ans de la CMU. Un bilan contrasté pour l'accès aux soins des migrants, Hommes et migrations, 1282, pp. 24-33.

MARCHE Hélène (2009) Le travail relationnel au cœur de l'expérience du cancer. Mises en forme ordinaires et institutionnelles de cette épreuve, Thèse de doctorat de sociologie, Université de Toulouse-le-Mirail.

MARIN Isabelle (2004) Migration et bonne mort, in Simone Pennec Éd., Des vivants et des morts. Des constructions de la "bonne mort », Université de Bretagne occidentale, ARS/Centre de recherche bretonne et celtique, pp. 165-168.

MARIN Isabelle (2007) Les rapatriements des malades en soins palliatifs, Liaison ASP, 36, pp. 19-22.

MBAYE Elhadji Mamadou (2009) La santé des immigrés en France : controverses autour d'un paradigme, Hommes et migrations, 1282, pp. 6-19.

MENORET Marie (1999) Les temps du cancer, Paris, CNRS, 237 p.

MISSAOUI Lamia (2010) Les réseaux familiaux transnationaux supports aux initiatives en matière de santé chez les Gitans catalans, Lien social et politique, 64, pp. 163-173.

MORILLON Anne, ETIEMBE Angeline, VEGLIC Patrick et FOLLIET Delphine (2007) Récit historique et mémoire de l'immigration en Bretagne, Tome 1, Rapport et Synthèse de l'étude Histoire et Mémoire de l'Immigration en Bretagne, consulté le 22 février 2012 [en ligne]. URL : http://www.odris.fr/documents.php

MOULLÉ François et DUHAMEL Sabine (Éds.) (2010) Frontières et santé. Genèses et maillage des réseaux transfrontaliers, Paris, L'Harmattan, $286 \mathrm{p}$.

OFPRA (2011) Rapport d'activité 2010, avril, 99 p.

PESTRE Élise (2010) La vie psychique des réfugiés, Paris, Payot, 318 p.

PIAN Anaik (2011) Les étrangers atteints de cancer en Bretagne : quelles difficultés, quelles ressources?, Communication au Colloque Le migrant est-il un patient comme les autres?, RVH 35, Quimper, 27 mai.

REGNARD Corinne (2009) La population étrangère résidant en France, Info migrations, 10, consulté le 7 septembre 2011 [en ligne]. URL : http://www.immigration.gouv.fr

RIMBERT Gérard (2007) Au secours des mourants : médicalisation et humanisation dans la gestion sociale de la mort, EspaceTemps.net, consulté le 6 septembre 2011 [en ligne]. URL : http://espacestemps.net/document3332.htlm 
SAKOYAN Juliette, MUSSO Sandrine et MULOT Stéphanie (2011) Quand la santé et les médecines circulent. Introduction au dossier thématique Médecines, mobilités et globalisation, Anthropologie et santé, 3, consulté le 30 janvier 2011 [en ligne]. URL : http://anthropologiesante.revue.org

SARRADON-ECK Aline (2004) Pour une anthropologie clinique : saisir le sens de l'expérience du cancer, in Patrick Ben Soussan Éd., Le cancer. Approche psychodynamique chez l'adulte, Eres, Ramonville St Agne, pp. 31-45.

STRAUSS Anselm (1992) La trame de la négociation. Sociologie qualitative et interactionniste, Textes réunis et présentés par Isabelle Baszanger, Paris, L'Harmattan, 311 p.

TAPIA Stéphane de (2005) Migrations et diasporas turques. Circulation migratoire et continuité territoriale (1954-2007), Maisonneuve et Larose, Paris, 402 p.

THOMAS Isabelle (2010) La régularisation pour raison médicale, conduite pratique à l'usage du médecin généraliste. Peut-on optimiser les démarches du médecin généraliste pour les demandes d'accès aux soins et de régularisation "étranger malade » au bénéficie de son patient "sans-papiers »?, Thèse de doctorat de médecine, Université de Grenoble, 221 p. 


\title{
De l'accès aux soins aux « trajectoires du mourir ». Les étrangers atteints de cancer face aux contraintes administratives
}

\begin{abstract}
Anaik Pian
Cet article s'intéresse d'une part, aux configurations d'entrée dans l'espace du soin des étrangers atteints de cancer dans trois villes bretonnes (Rennes, Brest et Quimper) et d'autre part, aux « trajectoires du mourir » en cas d'échec thérapeutique. Il insiste sur les obstacles administratifs rencontrés par ces patients selon les profils migratoires, du demandeur d'asile - en attente conjointe d'une régularisation pour soins - aux sans-papiers, en passant par les étrangers arrivés en France avec un visa touristique et dont le cancer a été diagnostiqué ou non au pays d'origine. La prise en charge oncologique ou palliative d'étrangers précaires confronte les professionnels à des « cas limites » qui interrogent des pratiques et des normes, qui plus est dans une région à faible immigration. Les logiques d'acteurs qui en résultent sont à replacer dans le contexte structurel pesant, en amont comme en aval, sur les parcours de soin et où se rencontrent politiques d'immigration et contraintes organisationnelles et financières des établissements de santé. Car si papiers et couverture médicale influencent les trajectoires de maladie, ils sont tout aussi importants dans les trajectoires du mourir.
\end{abstract}

\section{From Health Care Access to "End-of-life Trajectories". Foreign Cancer Patients Facing Administrative Obstacles}

\author{
Anaik Pian
}

This paper analyses the trajectories of cancer patients from non-EU countries in Brittany, at two separate moments: admission to medical care and end-of-life trajectories consequently to treatment failure. At both key moments, patients are confronted with various administrative obstacles according to their migration profile: from asylum seekers - who have conjointly asked for legalization on the basis of their health situation - over undocumented migrants, to foreigners who entered France with a tourist visa and whose cancer may or may not have been diagnosed previously in their country of origin. Providing cancer support services or palliative medical care for foreigners in a precarious social situation confront medical staff with border-line cases that question their practices and norms, and more especially so in Brittany, a region with low levels of immigration. As a consequence, the logics of actors must be understood in relation with the structural context that influence the beginning and the end of care trajectories, as health facilities that face structural organizational and financial constraints encounter those of immigration policies. Indeed, just as identity documents and possibilities of social protection influence the admission to medical care, they are of central importance for end-of-life trajectories. 


\section{Desde el acceso a la salud hasta las «trayectorias del morir»: los extranjeros enfermos de cáncer frente a los requerimientos administrativos}

\section{Anaik Pian}

Este artículo analiza, por un lado, las configuraciones de ingreso al espacio de cuidados de salud de los extranjeros enfermos de cáncer en Bretaña y, por otro lado, las «trayectorias del morir» en el caso de fracaso terapéutico. Se insiste sobre los obstáculos administrativos encontrados según el perfil migratorio, desde el demandante de asilo en espera de una regularización por cuidados de salud, pasando por los inmigrantes clandestinos hasta los extranjeros que llegaron a Francia con una visa turística y cuyo cáncer ha sido o no diagnosticado en el país de origen. La atención oncológica o paliativa de los extranjeros precarios confronta a los profesionales a casos «límite» que re-interrogan prácticas y normas, en particular en una región de baja inmigración. Las lógicas de actores observadas deben ser situadas en el contexto estructural sopesando los recorridos de cuidados de salud, y en dónde se re-encuentran políticas de inmigración y dificultades organizacionales y financieras de los establecimientos de salud. Si la posesión de papeles en regla y la cobertura médica influyen en las trayectorias de cuidados de salud, éstas son igualmente relevantes en las trayectorias del morir. 